\title{
Die heile Welt in der Werbung - Stereotype als Bestandteil von Werbestil
}

\author{
Martin Nielsen (Aarhus)
}

\begin{abstract}
Advertising is an area that has to rely heavily on reduced and simplified message content due to its often very short perception time or its high reception speed (cf. Kroeber-Riel et al. 2009). Therefore, advertising is a fertile field for investigating stereotypes whose reduced form of presentation serves a quicker orientation in an increasingly complex media society with stimulus overload (cf. Müller/Gelbrich 2014a). In this article, the principle of the "unspoiled world", "Die heile Welt", in advertising (cf. Thomsen 1998; Schoenen 2008; Siegert/Brecheis 2010) as a stereotype is identified and discussed. This particular advertising style has previously only been mentioned sporadically in literature. Therefore, in this article different case studies from German advertising and their scholarly thematisation (cf. e. g. Moritz 1997; Bohnsack 2003) are discussed from different perspectives. The specific stereotype advertising phenomenon of "heile Welt" is used to support, develop and differentiate different conceptualisations of advertising style.
\end{abstract}

\section{Einführung: gesellschaftliche und wirtschaftliche Rahmenbedingungen}

Es dürfte unstrittig sein, dass sich die Lebenswelt der meisten Menschen als außerordentlich komplex darstellt und dass sich der Alltag der Menschen - sei es als Bürgerinnen und Bürger, sei es als Konsumentinnen und Konsumenten - in einer schnelllebigen und durch permanente multimediale Informationsangebote geprägten Welt vollzieht. Aus wirtschaftlicher Sicht herrschen in fast allen Bereichen Marktbedingungen, bei denen Unternehmen unter einem sehr starken Wettbewerbsdruck stehen. Dieser Wettbewerb ist nicht länger nur ein Wettbewerb um Marktanteile und um Absatz, sondern jetzt in stärkerem Maße auch ein Aufmerksamkeitswettbewerb (Siegert/Brecheis 2010: 95-98). Unter solchen Marktbedingungen entfaltet sich ein Werbedruck der einzelnen Unternehmen, der in seiner Gesamtheit zur einer Werbeüberflutung, Informationsüberflutung und Reizüberflutung führt (cf. Müller 1997; Kroeber-Riel et al. 2009; Backhaus/Voeth 2010; Müller/Gelbrich 2014a). ${ }^{1}$

In einer solchen Situation sind die Rezipienten von Werbung (Information, Stimuli) in noch höherem Grad auf Rezeptionsstrategien angewiesen als dies in weniger werbeüberfluteten Zeiten der Fall war. Diese Reiz-, Informations- und Werbeüberflutung wird vor allem durch

\footnotetext{
${ }^{1}$ Durch Globalisierung, Internet und soziale Medien haben diese Tendenzen noch an Intensität zugenommen.
}

Linguistik online 79, 5/16 - http://dx.doi.org/10.13092/lo.79.3345

CC by 3.0 
selektive Wahrnehmung in der Form kurzer und kürzester Betrachtungszeiten (cf. KroeberRiel et al. 2009), Zappen, Verkürzung, Simplifizierung und Stereotypisierung zu bewältigen versucht (cf. z. B. Motschenbacher 2006; Femers 2007). Dabei sind vor allem die selektive und reduzierte Rezeption sowie die Herausbildung von Stereotypen ein probates Mittel zur Bewältigung dieser komplexen kognitiven Rezeptionsprozesse (cf. dazu unten Abschnitt 2).

Doch nicht nur die Rezipienten von Werbung, auch die Produzenten von Werbung entwickeln kommunikative Strategien, um den Herausforderungen des Aufmerksamkeitswettbewerbs zu begegnen. Eine produzentenseitige Strategie ist die simple Erhöhung des Werbedrucks. Um sich in der Kakophonie werblicher Kommunikation Gehör zu verschaffen, können Unternehmen noch häufiger, auf noch größeren Flächen, noch lauter und noch bunter kommunizieren. Diese Strategie entspringt einer intuitiven Logik des Verdrängungswettbewerbs auf dem Aufmerksamkeitsmarkt, birgt allerdings auch einen eigentümlichen Widerspruch in sich, dem sich die werbetreibende Wirtschaft offensichtlich nicht widersetzen kann: Der Versuch der Durchbrechung der Werbeüberflutung durch häufigeres, lauteres oder schrilleres Kommunizieren trägt zur Schaffung bzw. Verstärkung von Werbe-, Informations- und Reizüberflutung überhaupt erst bei. Das Problem, das es für die Unternehmen zu lösen gilt, wird durch die Problemlösungsmaßnahmen mit anderen Worten nur noch größer (Christensen/Cheney 2000: 249).

Eine andere Strategie von Textproduzenten im Aufmerksamkeitswettbewerb ist es, Werbung unterhaltsam zu verpacken. Als „Dank“ bzw. als Mehrwert für Zeit und Aufmerksamkeit der Rezipienten und deren Bereitschaft, sich mit einem Werbekommunikat auseinander zu setzen, bietet das werbetreibende Unternehmen kurzweilige, unterhaltsame und vergnügliche Werbung an (Infotainment, cf. z. B. Nielsen 2007).

Schließlich ist eine weitere mögliche Strategie von Produzenten der bewusste Einsatz von Stereotypen bzw. die Bedienung von Stereotypen, die bei den Rezipienten vorhanden sind. Gerade wegen der schnellen Erkennbarkeit, Identifizierung und Orientierung kommt der Gebrauch von Stereotypen den Rezeptionsstrategien der Zielgruppen entgegen und wird entsprechend von den Produzenten eingesetzt (cf. Nielsen 2016).

Vor diesem Hintergrund scheint die Beschäftigung mit Stereotypen aus Produzentensicht ein lohnender Ansatz, wenn Mechanismen der Stereotypisierung und Erklärungsansätze in Bezug auf Stereotype und stereotype Gestaltungsformen in der Werbung untersucht werden sollen. Das Ziel dieses Beitrags ist es daher, eine spezifische stereotype Form der Werbung, die heile Welt, zu erörtern und Erkenntnisse über diese Werbeform zu gewinnen, wobei das Augenmerk vor allem auf die Relation zwischen Stereotyp und Werbestil gerichtet wird. Die Untersuchung von Stereotypen in der Werbung, vor allem vom Stereotyp der heilen Welt, soll in diesem Beitrag vor allem dazu dienen, Konzeptualisierungen von Werbestil zu erläutern und zu differenzieren. Der methodische Ansatz ist dabei qualitativer Natur: Als explorative Studie versucht der Beitrag, ein bisher noch nicht systematisch untersuchtes Phänomen zu beschreiben. Der spezifische Werbestil ,heile Welt“" wurde bisher nur kurz und sehr sporadisch in der Literatur behandelt. In dem vorliegenden Beitrag soll er daher im Mittelpunkt des Interesses stehen. 
Der Beitrag gliedert sich folgendermaßen: Nach der kurzen Einführung und Darstellung gesellschaftlicher und wirtschaftlicher Rahmenbedingungen von Werbung (Abschnitt 1) folgen definitorische, typologische und funktionale Erörterungen zum Phänomen Stereotyp in der Werbung (Abschnitt 2). Anschließend werden unterschiedliche Konzeptualisierungen des Konstrukts Werbestil vorgestellt und erläutert und zum Stereotyp in Beziehung gesetzt (Abschnitt 3), wonach der spezifische stereotype Werbestil der heilen Welt dargestellt, exemplifiziert und erörtert wird (Abschnitt 4). Eine Thematisierung und Kritik der heilen Welt ist Gegenstand der anschließenden Ausführungen (Abschnitt 5). Den Abschluss bildet ein Ausblick auf interkulturelle Fragestellungen (Abschnitt 6).

\section{$2 \quad$ Stereotype in der Werbung}

Im Folgenden wird das Phänomen Stereotyp zunächst in seiner übergeordneten Funktion als generelle Orientierungshilfe dargestellt, bevor Stereotype als Charakteristikum der Werbung thematisiert werden.

\subsection{Stereotype als generelles kognitives Orientierungsangebot}

Geprägt hat den Terminus Stereotyp Walter Lippmann (1922) in seinem Buch Public opinion mit einer Metapher aus der Druckersprache, nach der Stereotype „,,fest miteinander verbundene Druckzeilen“ im Unterschied zu ,beweglichen Lettern““ bedeuten (Bußmann 2002: 650651). Im Grundsatz ist der Begriff des Stereotyps der Etymologie nach eine Verfestigung von an sich ursprünglich flexiblen Einheiten (cf. Kleinsteuber 1991: 60). Auslöser für die Entstehung von Stereotypen ist der Umstand, dass

wir uns - wohlgemerkt alle Menschen - gegen eine Welt von scheinbar überwältigender Kom-
plexität zur Wehr setzen: ,stereotypes as defense', wie es Lippmann formuliert. Da wir weder
Zeit noch Gelegenheit haben, uns intensiv und mit Details aus anderen Teilen der Welt ausei-
nanderzusetzen, entwerfen wir in unseren Köpfen verkürzte und reduzierte Bilder der Menschen
und ihres Lebens in anderen Kulturen. Nach Lippmann ist dies übrigens ein unvermeidlicher
und unausweichlicher Prozeß.

(Kleinsteuber 1991: 62)

Stereotype dienen mit anderen Worten der „Ökonomie der Wahrnehmung, der Strukturierung von Realität [...], da das menschliche Gehirn und seine Verarbeitungsmechanismen auf die verkürzte Abbildung angewiesen“ sind (Averbeck 2002: 274). Solche Formen der „Simplifizierung“, „Verallgemeinerungen“, „Vereinfachungen“ (Kleinsteuber 1991: 60) oder „Verkürzungen“ (Averbeck 2002: 274-275) sind somit „Mechanismen der Realitätsvereinfachung“ (Averbeck 2002: 275), mit der ,eine komplexe Wirklichkeit auf wenige, überschaubare und eingängige Andeutungen reduziert werden kann“(Kleinsteuber 1991: 60).

Damit scheint zumindest im sozial- und wahrnehmungspsychologischen Verständnis des Stereotypenkonzepts Konsens darüber zu herrschen, dass eine zentrale Funktion von Stereotypen die Orientierung in einer komplexen Umwelt, die Bewältigung von Informationsüberlastung 
und die rasche Erkennung und Bewertung von visuellen und verbalen Reizen sind (cf. z. B. Lippmann 1922, Kleinsteuber 1991; Six 19992; Müller/Gelbrich 2014a).

\subsection{Stereotype in der Werbung}

Diese Reduktionsfunktion - wir könnten auch sagen: Rezeptions- und Produktionsstrategie (cf. oben Abschnitt 1) - spielt aus den in Abschnitt 1 genannten Gründen insbesondere für die Werbung eine wichtige Rolle (Motschenbacher 2006: 57; Femers 2007: 41; Holtz-Bacha 2008; Siegert/Brecheis 2010: 243).

In der Werbung kommen Stereotype aus verschiedenen Lebensbereichen zum Einsatz. ${ }^{3}$ Zur Illustration der Verwendung unterschiedlicher Stereotype im werblichen Kontext seien nachfolgend einige der am häufigsten in der Werbung angewandten Stereotypen angeführt mit jeweiligen Belegen aus konkreten Werbekommunikaten:

Nationale Stereotype: Das Stereotyp des humorlosen Deutschen kommt z. B. in der Werbung für den Opel Karl zum Einsatz. In einem TV-Spot sagt die Kundin als Reaktion auf die Auflistung der Vorzüge des Fahrzeugs, einschließlich deutscher Ingenieurskunst, und angesichts des niedrigen Preises, dass das Angebot wohl ein Witz sein müsse. Daraufhin verblasst das Lächeln des Autoverkäufers und er antwortet, dass Deutsche keine Witze machen würden (cf. Opel 2015). ${ }^{4}$ Bei den Minzplätzchen der Marke After Eight kommt seit vielen Jahren der Slogan „die feine englische Art!“ (Nestlé s. a.) zum Einsatz (cf. Hars 2002: 93), der das nationale Stereotyp des aristokratischen Engländers sprachlich kondensiert.

Regionale Stereotype: Das regionale Stereotyp des unterkühlten und reservierten Norddeutschen wird z. B. bei der Werbung von Flensburger Pilsener relativ kontinuierlich verwendet. Beispielsweise verhält sich die Lokalbevölkerung vor allem auswärtigen Besuchern gegenüber in mehreren TV-Spots ausgesprochen wortkarg. In einem TV-Spot für Flensburger Pilsener kommt ein urbaner Typ (auch dies eine stereotype Darstellung) sehr dynamisch im Cabriolet mit attraktiver Freundin und Surfbrett an die Küste und erkundigt sich bei drei Männern, die vor ihrem Haus sitzen, nach Surfmöglichkeiten. Als er auf seine Anfrage nur Schweigen erntet, versucht er es noch in zwei anderen Sprachen, ebenfalls erfolgslos, ehe er wieder davonfährt. Nach seiner Abfahrt erfolgt folgendes kurze Gespräch unter den dreien:

(1) „Mann, der hatte ja 'n dolles Auto. Und "ne Menge Sprachen konnte der auch.“ „Aber genützt hat's ihm nichts“" (Flensburger 2011).

Das Unternehmen Flensburger versprachlicht und transportiert im Übrigen selbst dieses Stereotyp und dessen Anwendung in der Werbung auf seiner Website:

\footnotetext{
${ }^{2}$ Demnach sollen Stereotype (und Vorurteile, um die es Six hierbei geht) ,[...] einer effizienten Orientierung im sozialen Alltag dienen, die es erlaubt, möglichst schnell aus den angebotenen Informationen Erklärungen, Schlußfolgerungen und Vorhersagen zu machen [...]“(Six 1999: 829).

${ }^{3}$ Stereotype werden spätestens seit Ende des 19. Jahrhunderts in der Wirtschaftswerbung eingesetzt (cf. Reinhardt 1993). Insbesondere das Frauenbild wurde unter verschiedenen Vorzeichen immer wieder stereotyp dargestellt. So finden sich bei Reinhardt (1993: 411-412) Beispiele für ,das konservative Frauenbild der wilhelminischen Zeit“, „,das Motiv der emanzipierten Frau“ und „,das reaktionäre nationalsozialistische Frauenbild“.

${ }^{4}$ Autoverkäufer: „Our brand new car: There is plenty of room, five doors, six airbags, hill-start assist, all German engineered.“ Kundin: „At that price? That must be a joke!“ Autoverkäufer: „Germans don’t make jokes.“ (Opel 2015).
} 
(2) „Hier in Norddeutschland. Wo die Menschen dem Wunder der Sprache noch mit Respekt begegnen und jedwede Kommunikationsform höchst ökonomisch gestalten“ (Flensburger Brauerei s. a.).

Geschlechterstereotype: Weitgehend auf ihre Rolle als Hausfrau reduziert, deren größte Sorge der Reinigungsstandard beim Spülen zu sein scheint, wird die Frau in einem Spot für das Reinigungsmittel Sidol von Henkel auf YouTube:

(3) „Am Wochenende hatte ich meine Freundinnen zum Kaffeeklatsch zu Besuch. Der Tisch war schön dekoriert, wenn da nur nicht dies hässlichen schwarzen Streifen am Boden der Tassen gewesen wären [...]“ (Henkel Lifetimes 2015).

Dieses Beispiel zeigt neben der stereotypen (und nicht unbedingt realitätskonformen) Darstellung des modernen Hausfrauenalltags, dass die lange Tradition des stereotypen Frauenbildes bis in neue Marketinginstrumente, Medienformate und Textsorten ungebrochen anhält: Es handelt sich hierbei um so genanntes Content Marketing, bei dem die Marke im Vergleich zur herkömmlichen Werbung viel weniger in Erscheinung tritt, und bei dem Medium ist nicht vom klassischen Massenmedium Fernsehen, sondern vom sozialen Medium YouTube die Rede, das die Konsumentin auf eigene Initiative aufruft.

Dreßler (2008) hat in einer Longitudinalstudie über ein halbes Jahrhundert Darstellungen von Männern in Anzeigen im Stern in Bezug auf Häufigkeit, äußeres Erscheinungsbild, Freizeit und Beruf sowie Männertyp untersucht. Dabei stellt sie über den Untersuchungszeitraum zwar durchaus Veränderungen im Männerbild fest, u. a. das Aufkommen von androgynen Männerdarstellungen, aber eben auch unterschiedliche Stereotype: Werbung und die Medien bilden „Männer und Frauen nicht einfach ab, sondern vermitteln konkrete stereotype Vorstellungen davon, wie Frauen und Männer sein sollen“ (Dreßler 2008: 125). In Bezug auf die äußere Darstellung sind z. B. Schlankheit und kurze Haare (ebd.: 128) oder in Bezug auf das außerberufliche Umfeld die Rolle als Ehemann oder Partner (ebd.: 130) Ausdruck eines maskulinen Geschlechterstereotyps. Konkret wird dies z. B. in der Werbung des Rasierklingenherstellers Gillette verkörpert, dessen Slogan „Für das Beste im Mann“ lautet (Hars 2002: 154-155).

Berufsstereotype: Die stereotype Darstellung des Arztes, Zahnarztes oder Wissenschaftlers trägt in der Werbung zur Bildung und Weitertradierung eines Berufsstereotyps bei. Stereotyp wird die Inszenierung des Berufsstandes dabei vor allem durch die so gut wie ausschließliche Verkörperung dieser Stereotype durch seriös dreinschauende Männer mittleren Alters im weißen Kittel. Als Paradebeispiel für dieses Berufsstereotyp dürfte die Werbung für die Zahnbürste der Marke Dr. Best gelten, bei der ein älterer Herr im weißen Kittel die Vorzüge der schonenden Zahnbürste demonstriert (Dr. BEST s. a.; Hars 2002: 96-97). ${ }^{5}$

Insbesondere Stereotype, die sich auf eingefahrene Geschlechterrollen beziehen, sind in der Werbung zu beobachten. Auch die Wissenschaft hat sich bevorzugt vor allem mit dem Frauenbild in der Werbung befasst (Motschenbacher 2006, 2008; Fröhlich 2008; Schoenen 2008; Vennemann/Holtz-Bacha 2008; Wilk 2008).

\footnotetext{
5 Dass in diesem speziellen Fall der Kommunikator der Marke im Spot nicht aus einer Modellagentur kam, sondern tatsächlich (James) Best hieß, einen Doktortitel hatte und Professor für Dentalmedizin war (cf. Hars 2002: 97), tut der Aussagekraft des Beispiels keinen Abbruch.
} 
Vielleicht ist es der außerordentlich großen Dynamik, Komplexität und Vielschichtigkeit des Phänomens Geschlecht geschuldet, dass gerade dieser Themenkomplex so starken Stereotypisierungen unterworfen ist: Der Bedarf an „Realitätsvereinfachung“ (Averbeck 2002: 275) ist hier u. U. besonders hoch. Dabei kommen stereotype Auffassungen von Geschlechterrollen in der Werbung in Schemata oder situationellen Stereotypen zum Ausdruck, die sich nicht in Einzelstimuli und Schlüsselbegriffen erschöpfen, sondern sich vielmehr in Texten, Szenarien oder „Welten“ manifestieren. Ein solches stereotypes Szenario oder eine solche stereotype „Welt" ist eben die heile Welt in der Werbung (cf. unten Abschnitt 3 und 4).

Auch in der diachronen Sicht findet sich ein stereotypes Frauenbild ,in klischeehaft überzogener Darstellung [...], wobei auf eine realistische Darstellung des Hausfrauenalltags zugunsten von idealistischen Wunschbildern verzichtet wurde" (Reinhardt 1993: 399; cf. zum Frauenbild in der Werbung aus diachroner Perspektive insgesamt Reinhardt 1993: 397-414). Die heile Welt als strategische und gestalterische Praxis greift also auf eine lange Tradition stereotyper Darstellungen zurück.

Eine solche „Schablone“ hat zunächst eine „kognitiv-ökonomische“ (Femers 2007: 42) Funktion und bringt damit Rezipienteninteressen (schnelle Orientierung, rezeptive Vereinfachung komplexen Sachverhaltes) und Produzenteninteressen (schnelle Vermittlung, überschaubare und reduzierte Darstellung komplexen Sachverhaltes) scheinbar widerspruchsfrei zusammen.

Die Erforschung von Stereotypen in der Werbung, vor allem Geschlechterstereotypen, erfolgt in der Regel unter einem (gesellschafts)kritischem Blickwinkel, wie ihn u. a. Bendel (2008) speziell für die (text-)linguistische Werbeanalyse einfordert.

Dass dabei Stereotype als undifferenzierte Denkschemata und Denkbilder unhinterfragt weitertradiert werden, nimmt die Literatur in der Regel zum Anlass, solche Stereotype überhaupt zu untersuchen. Diese politisierende und bisweilen polemische Fragestellung hat einen sehr großen gesellschaftlichen Wert, sie soll in diesem Beitrag aber der eher technischen Perspektive weichen, in welcher Weise das Stereotyp der heilen Welt eingesetzt wird und wie es als Manifestation eines spezifischen Werbestils einzuschätzen ist. Die kritische Perspektive auf Stereotype in der Werbung, spezifisch bezogen auf das Stereotyp der heilen Welt, wird dafür in Abschnitt 5 noch einmal aufgegriffen.

\section{$3 \quad$ Konstrukt Werbestil und Stereotyp}

Werbestil ist ein häufig und oft undifferenziert verwendeter Terminus, der als Oberbegriff für die Gestaltungsmerkmale von Werbung angesehen werden kann. Es lassen sich daher unterschiedliche Konzeptionen vom Konstrukt Werbestil unterscheiden (cf. Kloss 2001a; Hoffmann 1999, 2012; de Mooij 2010). Ich spreche im Folgenden daher nicht von einer Werbestilkonzeption, sondern von mehreren. Für die verschiedenen Konzeptualisierungen von Werbestil spielen Stereotype jeweils eine unterschiedliche Rolle. Im Einzelnen lassen sich (mindestens) die folgenden fünf Werbestilkonzepte unterscheiden. Die Beispiele, die im Folgenden zur Veranschaulichung der jeweiligen Werbestilkonzeption aufgeführt werden, repräsentieren unterschiedliche Textsorten und nicht nur Werbeanzeigen, um die Gültigkeit etwas umfassender zu dokumentieren. 


\subsection{Werbestil als allgemeines Textsortenmerkmal}

Zunächst einmal kann Werbestil übergeordnet als ein allgemeines Textsorten- bzw. Diskursmerkmal konzipiert werden: „Es gibt typische Werbetexte bzw. einen typischen Werbestil““ (Hoffmann 2012: 179). Werbestil wäre dann gleichzusetzen mit dem Stil der Werbung schlechthin, also als varietätenlinguistisches Stilmerkmal, das generell für alle Werbetexte Gültigkeit besitzt. Werbestil kennzeichnet somit Werbung (Schweiger/Schrattenecker 2001), Werbekommunikation (Janich 2012) oder Werbesprache (Janich 2013) als solche (Hoffmann 1999, 2012). Da es sich dabei um eine sehr übergeordnete Konzeptualisierung handelt, spricht Hoffmann (2012: 179) zunächst bewusst etwas vage von „Werbestilistischem“. Dieses „Werbestilistische“ ist jedoch auf zwei verschiedenen Ebenen angesiedelt: Zum einen auf der übergeordneten Ebene, um die es in hier zunächst gehen soll, und zum anderen auf einer spezifischeren Ebene, bei der das „Werbestilistische“ zur strategischen Differenzierung eingesetzt wird, um Produkte und Marken über die Kommunikation unverwechselbar auftreten zu lassen. Man kann Werbestil also „sowohl dem typischen Werbestil als auch den verschiedenen Werbestilen" zuordnen (zu den verschiedenen Werbestilen cf. die Werbestilkonzepte in den Abschnitten 3.2. bis 3.5 unten). Der typische Werbestil kommt z. B. in dem Gebrauch von direktiven Sprechakten, von Sprechakten des Anbietens, von positiven Konnotationen, von Umgangssprache und von Produktbeschreibungen zum Ausdruck (cf. Abbildung 1). Dieses übergeordnete Verständnis von Werbestil und die Kenntnis davon dürfte sich mit dem in der allgemeinen muttersprachlichen Sozialisation erworbenen Textsorten- und Textmusterwissen des durchschnittlichen Sprechers decken, der Werbetexte (eben aufgrund des Werbestils) sehr schnell und sehr sicher als Werbetext einordnen kann. ${ }^{6}$

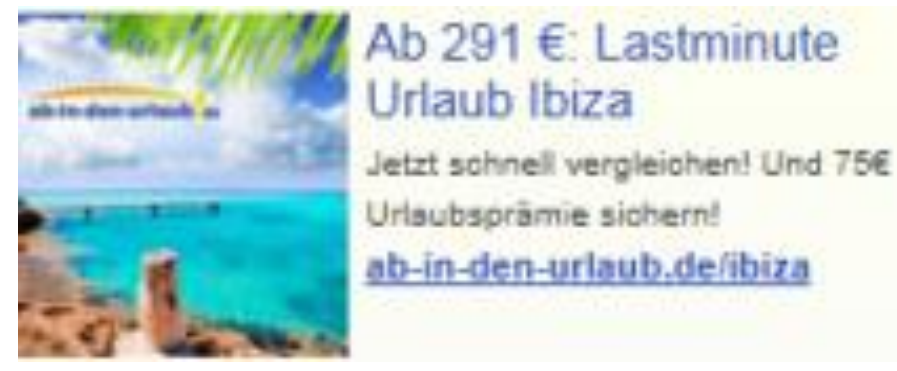

Abbildung 1: Werbestil generell (Bannerwerbung für ab-in-den-urlaub.de, fluege.de s. a.)

In diesem Verständnis von Werbestil spielen Stereotype eine eher untergeordnete Rolle. Zwar sind Stereotype in der Werbung generell nicht ungewöhnlich, aber es gibt selbstverständlich eine Vielzahl von Werbekommunikaten, in denen Stereotype nicht vorkommen.

\subsection{Werbestil als Paradigmatik von Gestaltungsweisen}

Werbestil kann auch als ein Stil aufgefasst werden, der unterschiedliche Gestaltungsweisen darstellt. Diese Auffassung von Werbestil kann als Bezeichnung für Klassen von Werbegestaltungen gelten (cf. Siegert/Brecheis 2010: 227-228; de Mooij 2010: 245-265), die in ihrer

\footnotetext{
${ }^{6}$ Cf. dazu auch den Begriff der persuasion knowledge, also des Bewusstseins darüber, dass man als Rezipient einem persuasiven, werblichen Text ausgesetzt ist und wie man sich angesichts des Wissens um die Persuasionsabsicht des Textproduzenten mit dem Text auseinandersetzt und Interpretationen des Textes und Auffassungen über den Textproduzenten formt (cf. Friestad/Wright 1994).
} 
Form eine Musterhaftigkeit aufweisen, die u. a. de Mooij (2010) dazu veranlasst hat, diese Art von Werbestilen nationenübergreifend zu Gruppen zusammenzufassen (cf. Tabelle1).

\begin{tabular}{|l|l|}
\hline Basic Form & Subcategories \\
\hline 1. Announcement & 1.1 Pure display \\
\hline & 1.2 Product message \\
\hline & 1.3 Corporate presentation, documentary \\
\hline 2. Association transfer & 2.1 Lifestyle \\
\hline & 2.2 Metaphor \\
\hline & 2.3 Metonymy \\
\hline & 2.4 Celebrity transfer \\
\hline 3. Lesson & 3.1 Presenter \\
\hline & 3.2 Testimonial/endorsement \\
\hline & 3.3 Demonstration \\
\hline & 3.4 Comparison \\
\hline & 3.5 „How to“ \\
\hline 4. Drama & 4.1 Slice of life \\
\hline & 4.2 Problem-solution \\
\hline & 4.3 Vignettes \\
\hline & 4.4 Theatre \\
\hline 5. Entertainment & 5.1 Humor \\
\hline & 5.2. Play or act around product \\
\hline 6. Imagination & 6.1 Cartoons \\
\hline & 6.2 Film properties in action \\
\hline & 6.3 Other, unrealistic acts \\
\hline 7. Special effects & 7.1 Product in action, animation \\
\hline & 7.2 Film, video techniques, artistic stimuli \\
\hline
\end{tabular}

Tabelle 1: Sieben Grundformen der Werbung (cf. de Mooij 2010: 247) ${ }^{7}$

Diese Art von Werbestil wird bisweilen auch als „executional style“ (de Mooij 2010: 245) oder „Tonalität“ (Schweiger/Schrattenecker 2001: 197; Müller/Gelbrich 2014b: 392) bezeichnet. De Mooij meint mit dieser Typologie sämtliche Werbeformen klassifizieren zu können. Allerdings bleiben die Systematik und Stringenz zweifelhaft, da - wie de Mooij selbst konzediert - in sehr vielen Fällen eine Mehrfachzuordnung eines gegebenen Werbekommunikats möglich ist. Dies ist vor allem auf die stark heterogenen Kriterien der Klassifikation zurückzuführen, die u. a. die Art der Produktpräsentation, die dargestellten Identifikationsressour-

\footnotetext{
${ }^{7}$ Bei de Mooij (1998: 242) findet man noch acht statt sieben Grundformen („2. Display“ eigene Grundkategorie) und 26 statt 23 Unterkategorien („,1.2 Factual explanation“, „4.5 Analogy“, „4.7 Dramatized lesson“).
} 
cen, die Kommunikatoren, die Qualität der Narrativität und die technisch-visuelle Darstellungsform umfassen. Trotz der mangelnden Systematik bietet die Klassifikation von de Mooij eine vor allem praktische Orientierungshilfe zur Bestimmung des Werbestils. Bei einem prototypischen Klassifikationsansatz, der bei de Mooij allerdings nicht thematisiert wird, statt bei einem aristotelischen Klassifikationsansatz der notwendigen und hinreichenden Bedingungen für die Kategorisierung, gewönne die Typologie an Gültigkeit. Beispielsweise vereint die heile Welt als Werbestil mehrere der in der Klassifikation aufgeführten Elemente in sich und ließe sich sowohl unter „Association transfer/Lifestyle“ als auch unter „Drama/Slice of life“ einordnen.

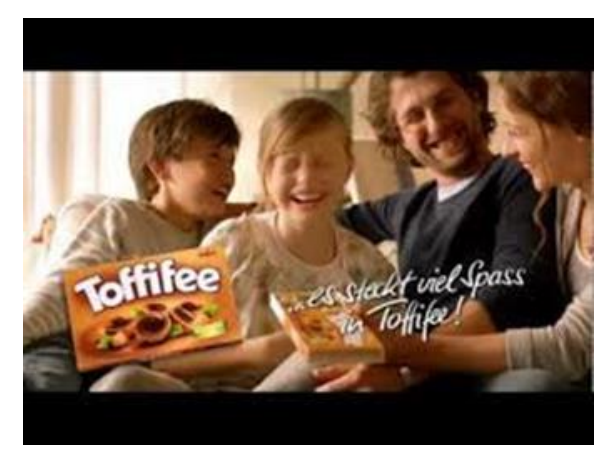

Abbildung 2: Werbestil nach Gestaltungsweise (TV-Spot für Toffifee, Toffifee 2012)

Unter der Grundform Assoziationstransfer versteht de Mooij (2010: 249) den Umstand, dass ein Produkt mit einem anderen Objekt, einer Person, einer Situation oder einem Umfeld - hier könnten wir auch sagen einer Welt - verbunden wird. In der Subkategorie „Lifestyle“ geht es speziell um den Assoziationstransfer in Bezug auf Menschen (de Mooij 2010: 250). Die Grundform „Drama“ definiert de Mooij (2010: 260) als Interaktion zwischen zwei oder mehreren Personen, die eine narrative Struktur aufweist. Die Subkategorie "Slice of Life“ ist durch einen dramatisierten Dialog und durch eine Alltagssituation aus dem ,echten Leben“ gekennzeichnet, in der dem Produkt eine zentrale Rolle zugewiesen wird. Bei dieser Version von Werbestil, vor allem bei den eben genannten Grundformen und Subkategorien in der Taxonomie von de Mooij, kommt Stereotypen eine zentrale Bedeutung zu. Gerade für „Lifestyle“ und „Slice of Life“ sind stereotype Vorstellungen von entscheidender Bedeutung, wenn nicht sogar konstitutiv.

\subsection{Werbestil als nationalkulturelles Unterscheidungsmerkmal von Werbung}

Während nach de Mooijs Ansicht alle Erscheinungen von Werbung in ihre Typologie eingeordnet werden können und die Typizität bzw. die Häufigkeit bestimmter Werbestile nationalkulturell bestimmt ist, lässt sich Werbestil jedoch auch generell am Kriterium Nationalkultur festmachen. Das Verständnis von Werbestil in diesem Sinne vertritt Ingomar Kloss unter dem Prädikat „Advertising Style“ (Kloss 2001a, 2001b). Nationaler Werbestil ist somit ein länderspezifischer Werbestil. Ungeklärt ist dabei jedoch, ob bei der Bestimmung der Variable ,Land' das Erscheinen der Werbung in den Medien des jeweiligen Landes, die Herkunft des werbenden Unternehmens bzw. dessen Marke oder etwa die Herkunft der verantwortlichen Werbeagentur das heranzuziehende Kriterium ist. Bei Kloss (2001a) ist das Erscheinen der Werbung im betreffenden Land der Ausgangspunkt: ,[...] media behavior is related to the communication styles, there are extremely wide differences in the advertising condition per 
country“ (Kloss 2001a: VII). De Mooij (2001: 20) spricht ebenfalls von den ,advertising styles of the USA and the countries of north-west Europe“. Konkret ordnet de Mooij spezifische Werbeformen (cf. oben Abschnitt 3.2) bestimmten Ländern bzw. Kulturen zu, deren Merkmale nach den Kulturdimensionen Hofstedes definiert werden. So ist nach de Mooij beispielsweise die japanische Kultur durch Kollektivismus, hohe Unsicherheitsvermeidung und große Machtdistanz gekennzeichnet, was sich in indirekter und weniger anstoßender Werbung manifestiert: „,The preference for entertainment as an advertising form in Japan is an example of this communication style“ (de Mooij 2001: 21). Das kommt u. a. in einem aus westeuropäischer Sicht graphisch unruhigen, überladenen oder gar chaotischen Werbestil zum Ausdruck (cf. Abbildung 3).

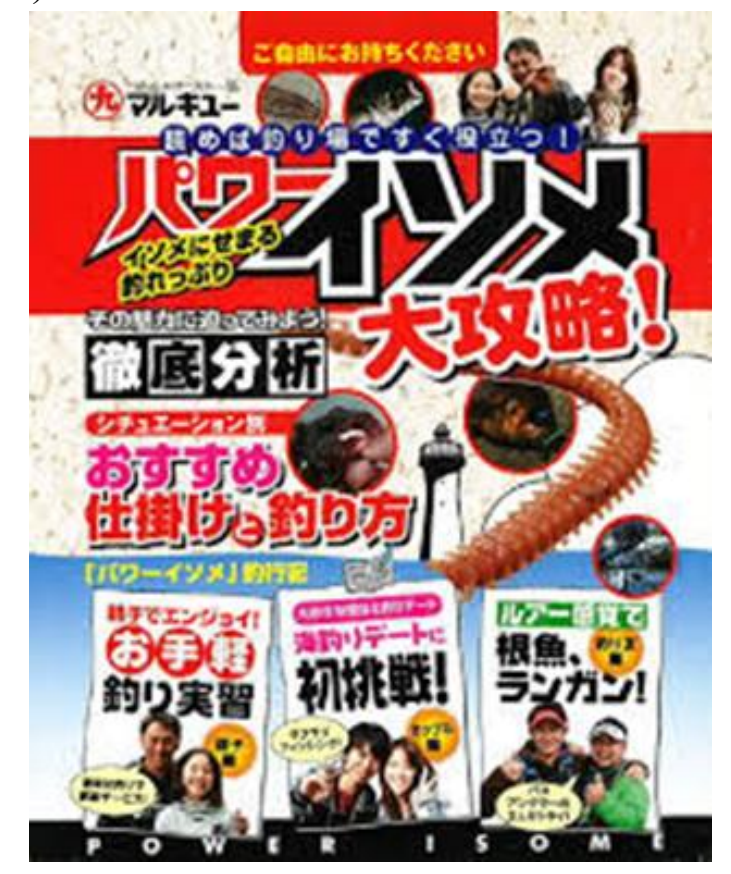

Abbildung 3: Werbestil national (Werbeanzeige für Marukya, Sachbücher für Angelzubehör, LRF 2012)

Ein wie auch immer gearteter deutscher Werbestil konnte sich in Deutschland lange Zeit nicht entwickeln: „Immer noch dominieren amerikanische Vorbilder, ein spezifisch nationaler Werbestil ist in der Werbung der sechziger Jahre nicht erkennbar“ (Schmidt/Spieß 1994a: 206). Anfang der siebziger Jahre ,gründet K. Gertner in Düsseldorf die GGK, deren Mitarbeiter [...] allmählich einen deutschen Werbestil zu entwickeln beginnen“ (Schmidt/Spieß 1994a: 216-217), eine Entwicklung, die bis in die achtziger Jahre dauerte (cf. Schmidt/Spieß 1994a: 238). Schmidt und Spieß machen mit anderen Worten den nationalen Werbestil an der Herkunft der Werbefachleute fest (cf. auch „Agenturstil“ (Schmidt/Spieß 1994a: 187)). Allerdings relativieren sie das Konzept des nationalen Werbestils, ,da Werbespots zu einem bestimmten Zeitpunkt nie einheitlich einem Stil folgen, sondern jeweils bis dato entwickelte Möglichkeiten zeitgleich realisieren“(Schmidt/Spieß 1994a: 237).

Die Rolle von Stereotypen in dieser nationalkulturellen Ausprägung von Werbestil ist nicht ganz eindeutig auszumachen. Einerseits lassen sich sicherlich etliche Fälle von Stereotypen identifizieren, die zu einem vermeintlich nationalkulturellen Werbestil beitragen, z. B. das Autostereotyp des fleißigen Deutschen oder das Heterostereotyp des ordnungsliebenden Deutschen, und u. U. auch das Stereotyp der heilen Welt (ob nun Auto- oder Heterostereotyp). Andererseits aber bedarf es interkultureller, sozusagen nationalkulturellübergreifender 
werbestilkontrastiver Untersuchungen, um den Einsatz solcher Stereotype als eindeutig spezifisch deutsch einordnen zu können.

\subsection{Werbestil als produkt(klassen)spezifisches Erkennungsmerkmal}

Ein weiterer Typus von Werbestil ist der produkttypische bzw. produktklassentypische Werbestil, wie ihn Janich (2013) beschreibt. Hier wird für unterschiedliche Produkte bzw. Produktklassen ein spezifischer oder typischer Werbestil angenommen. Dies wäre im Sinne von Hoffmann (2012) (cf. oben Abschnitt 3.1) ein differenzierender Werbestil, anhand dessen z. B. relativ eindeutig Kosmetikwerbung erkennbar ist (cf. Abbildung 4). Hier ist beispielsweise die blaue und weiße Farbe sowie die pseudowissenschaftliche Lexik stilbildend (cf. dazu auch Janich 2013: 219).

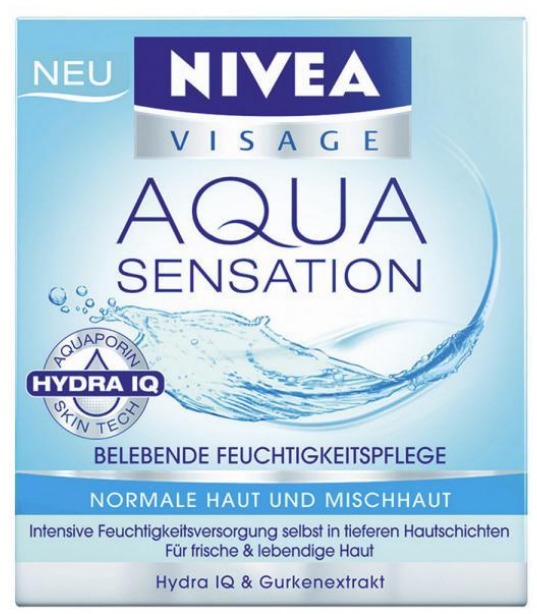

Abbildung 4: Werbestil produktklassentypisch (Verpackung von Nivea Visage Aqua Sensation, Amazon s. a.)

Auch in der produktklassenspezifischen Lesart von Werbestil kommen Stereotypen vor, z. B. das Berufsstereotyp des Arztes oder Wissenschaftlers im weißen Kittel (cf. dazu auch Abschnitt 2.2). Allerdings ist der Einsatz von Stereotypen hier nicht so stark an den Werbestil gebunden wie unter Abschnitt 3.2.

\subsection{Werbestil als unternehmens- oder markenspezifisches Erkennungsmerkmal}

In seiner konsequentesten Differenzierung ist Werbestil ein Merkmal, das ein Unternehmen bzw. eine Marke anhand seines Werbestils erkennbar werden lässt (cf. Schweiger/Schrattenecker 2001: 225-235; Siegert/Brecheis 2010; Müller/Gelbrich 2014a: 663-664). Da ein solcher Werbestil über viele Kontakte und damit erst nach längerer Zeit ${ }^{8}$ für den Rezipienten erlernbar und wiedererkennbar wird, erfordert ein unternehmens- oder markenspezifischer Werbestil Konsistenz über einen längeren Zeitraum, was wiederum in der Regel nur bei einer entsprechend langjährigen Zusammenarbeit mit einer Werbeagentur möglich ist.

\footnotetext{
${ }^{8}$ Damit ist ein solcher Werbestil im Prinzip nur über diachrone Studien erschließbar, cf. z. B. Reimann (2003) zur Werbung von Dallmayr oder Demarmels (2008) zur Werbung von Valserwasser.
} 


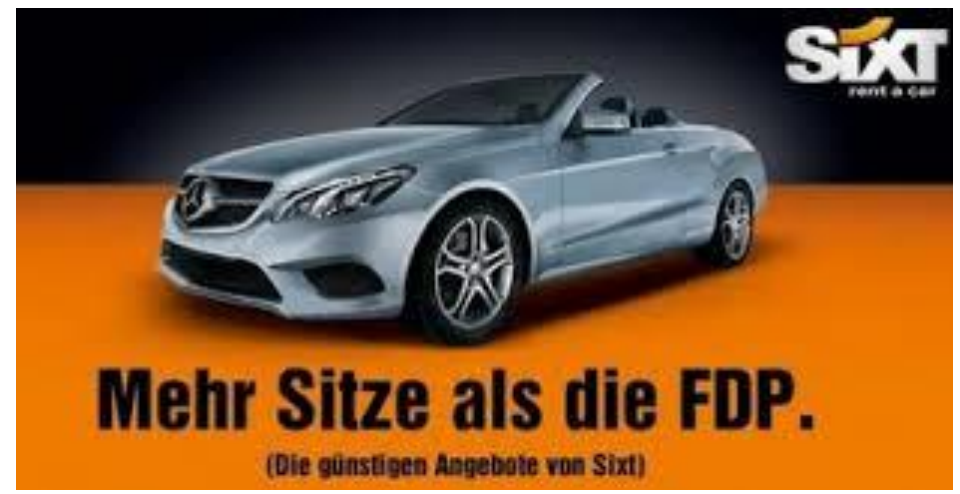

Abbildung 5: Werbestil unternehmensspezifisch (Plakat für Sixt, Saal 2013)

Das in Abbildung 5 dargestellte Plakat ist ein Beispiel für einen Werbestil mit sehr hohem Wiedererkennungswert. Der Autovermieter Sixt und die Werbeagentur Jung von Matt arbeiten seit über 15 Jahren zusammen und haben gemeinsam einen unverwechselbaren Werbestil entwickelt, der visuell im schwarz-weißen Buchstabendesign auf orangenem Hintergrund zum Ausdruck kommt. Verbal ist er gekennzeichnet durch kurze, prägnante Texte und entfaltet seinen originellen Humor aus der Text-Bild-Relation, indem er sich gleichzeitig auf das aktuelle Zeitgeschehen bezieht.

Die Rolle von Stereotypen in dieser Auslegung von Werbestil ist wie auch in Abschnitt 3.4 beschränkt, weil diese Werbestilkonzeption eher idiosynkratrisch an eine spezifische (Unternehmens-)Marke gebunden ist. Die individuelle Ausprägung des Werbestils ist durch das Unternehmen und die Werbeagentur frei gestaltbar und setzt in viel höherem Maße auf die Differenzierbarkeit und Unverwechselbarkeit und wird sich folglich eher selten stereotyper Darstellungen bedienen (es sei den eine Marke besetzt ein Stereotyp), weil es ansonsten zu austauschbarer Werbung kommt.

\section{$4 \quad$ Ein stereotyper Werbestil: Die heile Welt}

Nachdem oben Stereotype in der Werbung dargestellt wurden und anschließend das Konstrukt Werbestil erörtert wurde, soll in einer Zusammenschau dieser beiden Konzepte nun ein stereotyper Werbestil vorgestellt und diskutiert werden. Der Werbestil der so genannten heilen Welt dürfte prinzipiell den meisten Konsumenten bekannt sein (wenn auch nicht unbedingt unter diesem Namen), da er wegen seiner langjährigen Nutzung in der Werbepraxis prinzipiell jedem im Laufe seiner massenmedialen Sozialisation wiederholt begegnet sein wird.

Zur Illustration des Phänomens sei zunächst auf Abbildung 6 verwiesen, in der in der werblichen Textsorte Plakat eine solche heile Welt dargestellt wird. ${ }^{9}$

\footnotetext{
${ }^{9} \mathrm{Cf}$. auch oben Abbildung 2, die zwar als Beispiel für Slice-of-Life-Werbung gebracht wurde, jedoch gleichzeitig ein Heile-Welt-Szenario darstellt.
} 


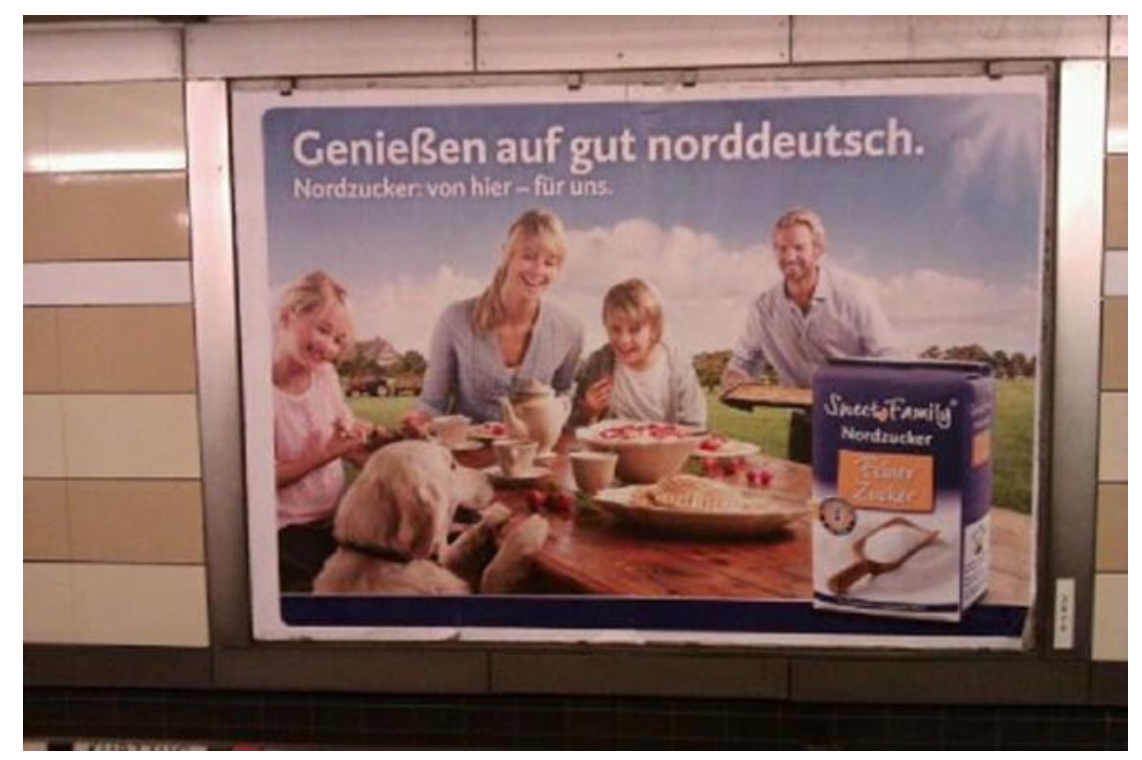

Abbildung 6: Heile-Welt-Werbung (Plakat der Firma Nordzucker an der U-Bahn-Haltestelle Hudtwalkerstraße in Hamburg. Foto: Martin Nielsen)

Als Denotat ist auf dem Plakat eine Familie mit Vater, Mutter, Tochter und Sohn sowie Hund zu sehen, die im Freien am Frühstückstisch sitzen, der u. a. mit Milchprodukten und Obst gedeckt ist. Im Vordergrund ist das Produkt, weißer Zucker der Marke Sweet Family der Firma Nordzucker abgebildet. Headline und Subheadline lauten „Genießen auf gut norddeutsch. Nordzucker: von hier - für uns.“ Konnotativ werden familiäre Harmonie, Zusammenhalt, Zufriedenheit, Freude am Genuss und Abwesenheit von Problemen und Dissonanzen ausgedrückt. ${ }^{10}$ Auf der Homepage des Unternehmens wird die heile Welt folgendermaßen versprachlicht:

(4) „Sweet Family für die süßen Augenblicke im Leben. Frühstück mit Freunden, Kindergeburtstage, Familienfeste, geruhsame Teestunden oder eine Cocktailparty. Das sind die süßen Augenblicke im Leben - Sweet Family gehört einfach dazu“ (Nordzucker s. a.).

Weitere Beispiele für den Werbestil der heilen Welt finden sich vor allem in der Lebensmittelindustrie, und hier insbesondere im Bereich Süßwaren. Das in Abschnitt 3.2 angeführte Beispiel von Toffifee, das dort vor allem als Illustration für die bildlich stereotype Darstellung der heilen Welt diente, kann hier noch einmal aufgegriffen werden, weil auch die sprachliche Gestaltung der heilen Welt in dem betreffenden Spot ein ausgesprochen stereotypes Bild der heilen Welt suggeriert. So lautet der Text des Werbesongs folgendermaßen:

(5) „Schenk‘ ein Lächeln, schenk‘ ein Lachen, und das Leben lacht mit dir! Teil‘ ein Lächeln und teil‘ die Freude, und das Glück, es lacht mit dir! Schenk‘ ein Lächeln, schenk' ein Lachen, und das Leben lacht mit dir! Denn in Toffifee steckt, was allen schmeckt!“

\footnotetext{
10 Dass der regionale Bezug textuell („norddeutsch“, „Nordzucker“, „,von hier“) und visuell (flache Landschaft) werbestrategisch hervorgehoben wird, ändert nichts an der Tatsache, dass das Szenario der heilen Welt hier vor allem visuell sehr stark in den Vordergrund tritt.
} 
Ähnliche stereotype Bilder einer heilen Welt werden in den Kampagnen von anderen Süßwarenherstellern vermittelt. In der Werbung für Kinderüberraschungseier werden die hoffnungsvollen Erwartungen von der treusorgenden Mutter erfüllt (cf. Kinder 2012); in der leicht nostalgischen Werbung für die Sahnebonbons von Werthers Echte wird das harmonische und liebevolle Verhältnis zwischen Opa und Enkel dargestellt (cf. Werthers 2012); Merci thematisiert in den Werbespots den Markennamen, indem das Thema Dankbarkeit über Generationsklüfte hinweg narrativ aufbereitet wird (cf. Merci 2015); und der Schokoladenproduzent Lindt entwirft in seiner aktuellen Osterkampagne die heile Welt der unbekümmerten Kindheit (cf. Lindt 2015).

Neben den oben angeführten zahlreichen empirischen Belegen für den Werbestil der heilen Welt lässt sich in Bezug auf diesen Werbestil feststellen, dass er auch in der theoretischen Literatur in vielen Fällen explizit thematisiert wird, ohne dass er jedoch Gegenstand einer Gesamtdarstellung wie in dem vorliegenden Beitrag gewesen wäre. Dabei ist die Tatsache, dass sich für diesen Werbestil ein Terminus durchgesetzt hat (cf. unten Abschnitt 5.2 zur Begriffsgeschichte), ein weiteres Indiz dafür, dass die Annahme eines solchen Werbestils gerechtfertigt ist.

Die Beschreibungen der heilen Welt durch unterschiedliche Forscherinnen und Forscher erfolgen oft aus der Perspektive der Stereotype in der Werbung. Die heile Welt stellt in der Regel eine Situation dar (cf. oben Abschnitt 3.2 Slice-of-Life), die von Harmonie, sozialem Glück und idyllischer Problemlosigkeit gekennzeichnet ist und die sich im Rahmen einer klassischen Kernfamilie oder Partnerschaft abspielt: „,Heile Welt ${ }^{\star}$, das impliziert intakte Natur, schöne, historische Bauten, sympathische, einander freundlich verbundene Menschen, traditionelle Familien, Harmonie und dgl. mehr“ (Thomsen 1998: 199) und „,Heile Welt“ operiert mit konservativen Wertskalen, und dazu gehören vor allem intakte Familien, Kinder und Haustiere“ (Thomsen 1998: 201). Integrativer Bestandteil der heilen Welt ist so gut wie immer die stereotype Geschlechterrolle der Frau als ,die fleißige Hausfrau, die fürsorgliche Mutter oder einfach die Frau, die die Idylle des Familienlebens in vollen Zügen genießt" (Vennemann/Holtz-Bacha 2008: 79). Zur heilen Welt gehört entsprechend auch, dass sie prinzipiell im bisweilen krassen Widerspruch zur sozialen Realität steht, denn „die Menschen der Werbewelten leben in einer heilen Welt, der Welt der glücklichen Menschen und den vielen Dingen, die man zum Glück braucht“" (Schoenen 2008: 34). Es ist denn auch diese Übertreibung und die komplette und konsequente Ausklammerung der tatsächlichen, für die meisten Menschen erfahrungsgemäß gegebenen Probleme und Herausforderungen des Lebensalltags, welche die recht umfassende Kritik an dem Werbestil der heilen Welt hat aufkommen lassen.

Die Erzählung von „Familienharmonie, Heile-Welt-Geschichten“ (Schmidt/Spieß 1994a: 227) und die Inszenierung von „Familie als Hort und Keimzelle der Gesellschaft“ (Schmidt/Spieß 1994a: 231) wird in der Narration von Fernsehspots besonders deutlich. Seeßlen hat eine Typologie von „Werbearten“ entwickelt, die über einem „Set von Werbedramaturgien operieren“ (Schmidt/Spieß 1994a: 235) und den Heile-Welt-Fernsehspot folgendermaßen benannt und definiert: 
Die Heim-und-Herd-Dramaturgie: Eine Gruppe, eine Familie, ein Teil einer Familie versammelt sich durch und um eine Ware und ihren Gebrauch zu inniger Gemeinschaftlichkeit [Hervorhebung im Original, MN].

(Schmidt/Spieß 1994a: 236)

Vor dem Hintergrund der obigen Ausführungen stellt sich die Frage, welchen der in Abschnitt 3 dargelegten Werbestilkonzeptionen der Werbestil ,heile Welt“" am ehesten zuzuordnen ist. Für die Einordnung des Werbestils ,heile Welt" als eine charakteristische Gestaltungsweise im Sinne von Abschnitt 3.2 spräche, dass die Klassifikation von de Mooij zwei potenzielle Kategorien bereithält, unten denen sich die heile Welt einordnen ließe: Zum einen könnte die heile Welt der Subkategorie „Lifestyle“ unter der Basisform „Association Transfer“ zuordnen lassen. In dem Fall wäre dann das traditionelle Familienmuster mit konservativer Rollenverteilung als ein Lebensstil anzusehen, der entsprechende Stereotype verkörpert und weitertradiert und in dem sich die Zielgruppe spiegeln kann. Eine andere, möglicherweise plausiblere Zuordnung wäre die Basisform „Drama“ mit der Subkategorie „Slice-of-Life“. Hier wird in stereotypischer Form ein Ausschnitt aus dem Alltagsleben präsentiert. Als Beispiel gibt de Mooij einen deutschen TV-Spot für die Küchenrollenmarke Bounty an, in dem durchaus auch die heile Welt - hier in der Form des gemütlichen Beisammenseins von Familie und Freunden beim Grillen - erkennbar ist.

Es ließe sich jedoch auch dafür argumentieren, dass der Werbestil der heilen Welt am ehesten der unter Abschnitt 3.4. beschriebenen Konzeptualisierung von Werbestil zugerechnet werden sollte, also ein produktklassentypischer Werbestil. Denn die heile Welt kommt offensichtlich bevorzugt gerade in Kampagnen für Süßwaren bzw. Schokoladenprodukte zum Einsatz. Allerdings ist die stereotype Darstellung der heilen Welt auch in benachbarten Produktkategorien zu beobachten. Frühstückscerealien wie Kelloggs Cornflakes oder auch die Margarinenmarke Rama zeichnen ebenfalls Bilder des glücklichen, gänzlich problemfreien Zusammenlebens der Kernfamilie.

\section{$5 \quad$ Thematisierung und Kritik der heilen Welt im deutschen Kontext}

Ein wesentlicher Grund für die Fokussierung der heilen Welt als Werbestil ist der Umstand, dass dieser Stil in der Forschungsliteratur - zum Teil kritisch - thematisiert wird. Im Folgenden werden daher einige zentrale Aspekte dieser Thematisierung vorgestellt und diskutiert.

\subsection{Geschichte}

Geschichtlich wird die Entstehung bzw. die Wiederentstehung des Werbestils der heilen Welt ${ }^{11}$ als eine Rückbesinnung auf traditionelle Werte der Vorkriegszeit betrachtet:

\footnotetext{
11 Der Begriff der heilen Welt stammt ursprünglich aus der Feder des deutsch-baltischen Dichters Werner Bergengruen (1892-1964), der in seinem Gedichtband Die heile Welt (1950) die Unverletzbarkeit innerer Werte angesichts der Katastrophen der äußeren Welt preist. Das seinerzeit sehr populäre Werk von Bergengruen wurde im Nachhinein bisweilen auch als Verdrängung von (vor allem durch nationalsozialistische Verbrechen ausgelöste) Not und Leiden gesehen. Die in der Werbung ebenfalls eskapistische Schönfärberei des Lebensalltags (unter Ausblendung der Schwierigkeiten des Alltagslebens und unter kompletter Ausklammerung der enormen Probleme der Vergangenheitsbewältigung) lud daher dazu ein, den Begriff der heilen Welt zweckentfremdet von der Literatur bzw. Poesie auf die Gebrauchstexte der Werbung zu übertragen.
} 
Konsum befriedigt nach dem Krieg das Bedürfnis nach Heile-Welt-Phantasien, in denen die Frau nur zwei Sorgen hat: was sie anziehen und was sie kochen soll. Das Alltagsidyll der Werbung wird oft als Indiz für die Vergangenheitsverdrängung in der Nachkriegszeit gewertet.

(cf. Wilk 2008: 66 sowie die dort angegebene Literatur)

Auch Siegert/Brecheis (2010) messen in ihrer Einschätzung der Entstehungsgeschichte des Werbestils der Nachkriegszeit große Bedeutung bei:

Wiederaufbau, Wirtschaftswunder und die ,heile Welt" in der Werbung [...] Inhaltlich versuchte die Werbung zunächst, sich auf das Private zu konzentrieren und knüpfte nahtlos und unter Ausklammern der Zeit des Nationalsozialismus an die Heile-Welt-Idylle der Vorkriegszeit an. Dazu gehörte auch, dass die gesellschaftliche Bedeutung der Frau sehr deutlich an Familie und Haushalt rückgebunden wurde.

(Siegert/Brecheis 2010: 74)

Der stereotype Werbestil der heilen Welt hat sicherlich in den 1950ern seine Blütezeit erlebt, erweist sich aber als durchaus langlebig. So lässt sich der Fortbestand des Werbestils der heilen Welt nicht nur anhand vorwissenschaftlicher Erfahrungswerte in der Form des regelmäßigen Antreffens solcher Werbekommunikate im Medienalltag belegen. Auch die Literatur bescheinigt dem Werbestil der heilen Welt, dass er sich jeweils Ende der 80er (Siegert/Breicheis 2010: 82), Ende der 90er (Thomsen 1998: 197, 201) und Ende des ersten Jahrzehnts des neuen Jahrtausends (Vennemann/Holtz-Bacha 2008: 79-80) in der Werbung immer noch identifizieren lässt.

\subsection{Eskapismus}

In der kritischen Sicht vor allem der medienwissenschaftlichen Literatur wird die eskapistische Dimension der heilen Welt moniert: ${ }^{12}$ „Die beschönigenden Heile-Welt-Szenarien [...] mit einer idealisierend-verklärten Darstellung von Wirklichkeit“" (Moritz 1997: 52) lädt den unbekümmerten Konsumenten und Bürger dazu ein, ,in die farbenfrohe, schöne heile Welt des Konsums einzutauchen und der alltäglichen Tristesse den Rücken zu kehren“ (Moritz 1997: 49). „Der Flanierende bewegt sich von der grausig-gruseligen Nachrichtenwelt in die heile Warenwelt der Kaufhäuser, in der er alles, nur nicht die Schattenseiten dieser Gesellschaft anzutreffen wünscht“ (Moritz 1997: 58). Diese Kritik, die auch bei Holtz-Bacha (2008), Vennemann/Holtz-Bacha (2008) und Schoenen (2008) laut wird, geht im Prinzip an den Unternehmen und Werbetreibenden vorbei: Sie machen sich lediglich die oben in Abschnitt 1 dargelegten Rezeptionsstrategien und kognitiven Mechanismen zunutze, die Rezipienten anwenden. Allerdings ist die Kritik insofern berechtigt, als dass sie zu einer sehr grundlegenden gesellschafts- und medienpolitischen Diskussion beiträgt, der sich die Werbenden nicht entziehen können, nämlich die Frage nach der Beziehung und der Kausalität zwischen Gesellschaft und Werbung: Beeinflusst die Werbung die sie umgebende Gesellschaft oder spiegelt Werbung lediglich bereits existierende gesellschaftliche Normen, Auffassungen und Interaktionsformen wider? (cf. Siegert/Brecheis 2010: 67-71).

\footnotetext{
12 Da hier vorzugsweise die synchrone Perspektive interessieren soll, habe ich auf eine systematische historische Übersicht der Kritik an der Werbung verzichtet. Zur gesellschaftlichen und ideologischen Kritik an der Konsumgesellschaft und der Werbung cf. u. a. Ruppert (2000: 766-767) und Gasteiger (2008).
} 


\subsection{Rezeption und Metakognition}

Eine pragmatische, wenn auch bequeme Auslegung der Konsequenzen von Heiler-WeltWerbung bietet Kloss:

Furthermore, advertising is reproached with showing an ,intact (idealized) world'. It is regarded as mendacious because it shows the world in a way that does not exist [...] It works with a form of exaggeration that is immediately recognizable as such. In Germany, the customer has meanwhile learned to handle advertising and to relativize it.

(Kloss 2001b: 138)

Seine Beurteilung, dass die deutschen Rezipientinnen und Rezipienten die heile Welt der Werbung durchaus als realitätsfern und übertrieben einzuschätzen und aufgeklärt damit umzugehen wissen, ist gewiss plausibel. Andererseits aber dürfen die durch diesen Werbestil oft propagierten Frauenrollen sicherlich nicht unterschätzt werden (cf. Wilk 2008).

Dieser offensichtlich aufgeklärten Gruppe von Konsumentinnen und Konsumenten ${ }^{13}$ stehen jedoch auch solche gegenüber, die Heile-Welt-Werbung wenn nicht für bare Münze nehmen so doch nicht unbedingt reflektiert-distanziert rezipieren ${ }^{14}$, sondern eher direkt als Identifikationsressource nutzen (cf. Schmidt/Spieß 1994a: 201). Auf jeden Fall werden diese Zielgruppen - auch wenn sie heute etwas kleiner sind, bleiben sie stabil und kauffreudig - immer noch durch solche Werbung angesprochen und erreicht (cf. oben Abschnitt 5.1).

\subsection{Intertextualität, Ästhetisierung, Ironisierung}

Das Stereotyp der heilen Welt ist also nach wie vor ein oft genutzter Werbestil in der Werbung. Ein weiterer Aspekt, der zur Darstellung des Werbestils der heilen Welt beiträgt, ist der intertextuelle Umgang mit dem Stereotyp. Ein Stereotyp darf auf jeden Fall spätestens dann als eine feste kognitive Größe angesehen werden, wenn intertextuell darauf angespielt werden kann. Generell hat Werbung schon seit Längerem Selbstreferenzialität als Wirkungsmittel genutzt. Es wird in diesem Zusammenhang nicht nur auf Zitate, syntaktische Strukturen, lexikalische oder visuelle Elemente aus werbefremden Referenztexten angespielt (wie z. B. Zitate aus Politik oder Literatur, Sprichwörter, Redensarten, Märchen oder andere literarische Textgattungen), sondern auch auf Referenztexte aus der Werbung selbst (cf. Janich 1997; Christensen 2001). ${ }^{15}$ Spezifisch für die „Alm, eine Landschaft, die in besonderer Weise als Stereotyp für eine natürliche und heile (Um-)Welt steht" (Bohnsack 2003: 117), hat Bohnsack (2003) eine Analyse eines Plakats durchgeführt, das die heile Welt ästhetisierend und ironisierend verfremdet (cf. Abbildung 7). Die „Ironisierung des Klischees von der heilen Welt der Alm und des an derartigen Klischees orientierten Lebensstils“ (Bohnsack 2003: 116) ist nur

\footnotetext{
${ }^{13}$ Cf. auch Siegert/Brecheis (2010: 82-83): „Besonders Jugendliche lernen früh, dass die Versprechungen der Werbung in der Wirklichkeit nicht erfüllt werden“.

${ }^{14}$ In den Anfängen der Fernsehwerbung standen Lernprozesse „des am Kinospot und der Printwerbung geschulten Konsumentenpublikums“ noch aus, ,das den in der Werbung gemachten Aussagen in erstaunlichem Umfang glaubt“" (Schmidt/Spieß 1994a: 201).

${ }^{15}$ Cf. dazu den sehr illustrativen Titel des Aufsatzes von Janich (1997): „Wenn Werbung mit Werbung Werbung macht ...".
} 
bei entsprechender Kenntnis dieser Klischees möglich, und die wiederum entsteht nur bei langjähriger, wiederholter Nutzung in der Werbung. ${ }^{16}$

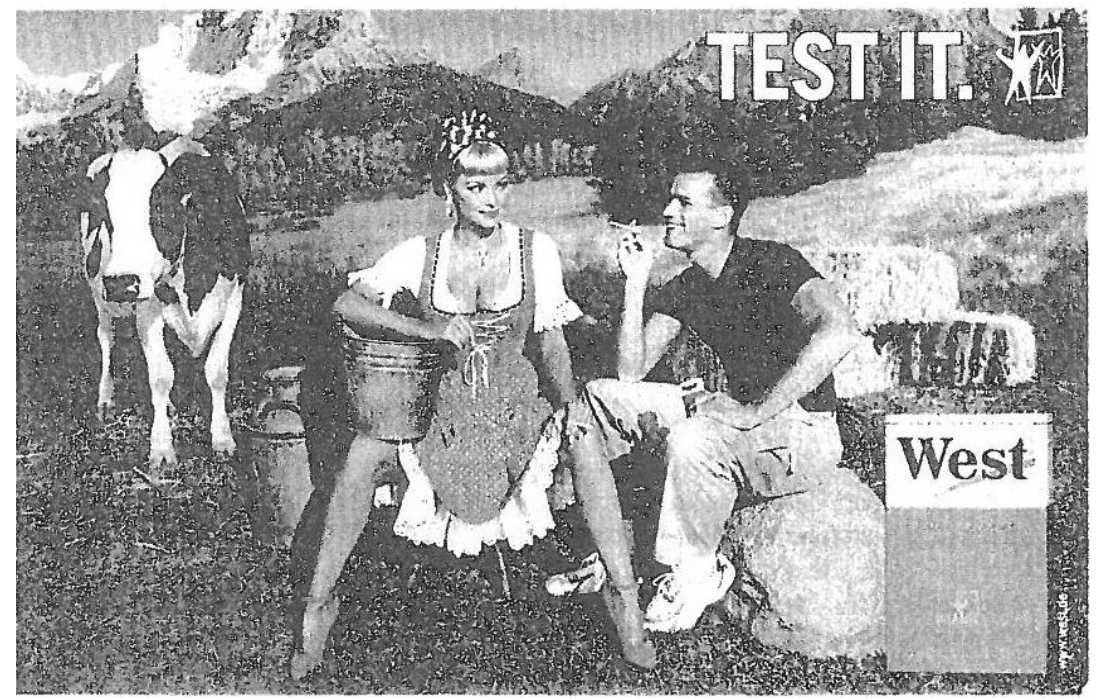

Abbildung 7: Ironisierung von Heile-Welt-Werbung (Plakat der Zigarettenmarke West, Bohnsack 2003: 110)

\section{Ausblick}

In den obigen Ausführungen zur heilen Welt sind interkulturelle Fragestellungen (zunächst) unberücksichtigt geblieben. Inwiefern heile Welt z. B. ein spezifisch deutsches Werbephänomen sein könnte, konnte im Rahmen dieses Beitrags nicht erörtert werden. Wie in Abschnitt 3.4 erwähnt würde dies systematische kulturübergreifende Studien erfordern, die an dieser Stelle nicht geleistet werden können.

Dennoch sollen abschließend einige interkulturelle Überlegungen angestellt werden, die sich vor allem auf die potenzielle Nationalspezifik des Werbestils der heilen Welt beziehen. In dem Zusammenhang sei kurz die Studie von Hindrichsen/Schulz (2004) vorgestellt. In dieser Masterarbeit wurden sechs dänisch synchronisierte TV-Spots (Ausgangssprache Deutsch) der Firma Storck (Campino Sahnebonbons, Toffifee, Storck Riesen, Merci) und der Firma Ferrero (kinder Überraschung, kinder Milch-Schnitte) untersucht. Nach einer semiotischen, rhetorischen, kulturellen und typologischen Analyse der Spots als manifeste Textprodukte, wurden die Spots auch einer Rezeptionsanalyse in dänischen Fokusgruppen unterzogen. Die Studie kommt zu dem Schluss, dass die dänischen Rezipienten wegen der Synchronisierung, wegen des Pathos und wegen der verkörperten Werte (,heile Welt") als nicht positiv auffassen. Gerade die realitätsferne und damit falsche Familienidylle scheint die Sympathien der Rezipienten $\mathrm{zu}$ verspielen. Inwiefern eine entsprechende deutsche Fokusgruppe die gleichen Antipathien hegt, geht aus dem Untersuchungsdesign nicht hervor. Allerdings sprechen mehrere Gründe dafür, dass die Spots bei einer deutschen Zielgruppe viel eher positiv aufgefasst würden. Zunächst einmal sind die Marken deutsch: Hinter vier der Marken steht Storck als deut-

\footnotetext{
16 Sicherlich hat dazu auch die im deutschen Sprachraum bekannte Marke Bärenmarke beigetragen, die konsequent die heile Welt in der Form der unzerstörten und idyllischen Natur der Alpenlandschaften seit vielen Jahrzehnten als visuelle Folie für Produktdarstellung, Markenaufbau und Markenpflege nutzt bzw. inszeniert.
} 
sches Unternehmen, und obwohl Ferrero ein italienisches Unternehmen ist, haben die Marken kinder Überraschung und kinder Milch-Schnitte ihren Ursprung in Deutschland, wo sie von der Mitte der 50er Jahre gegründeten deutschen Tochtergesellschaft entwickelt wurden. Ferner werden bei Storck alle TV-Spots in Deutschland mit deutschen Schauspielern gedreht und in Deutschland Prä- und Posttests unterzogen und gegebenenfalls geändert, wenn sie nicht in den Geschmack der deutschen Zielgruppe fallen. Für die ausländischen Märkte werden die Spots synchronisiert, allerdings ohne Prä- und Posttests und deshalb auch ohne etwaige Anpassung an den Geschmack der jeweiligen Zielgruppe (Hindrichsen/Schultz 2004: 18-20). Dieser Untersuchung zufolge gibt es also Hinweise darauf, dass der Werbestil der heilen Welt ein spezifisch deutscher Werbestil sein könnte. In dem Fall käme auch die Werbstilkonzeption aus Abschnitt 3.3 in Frage: ein nationalkultureller Werbestil. Allerdings ist dabei zu beachten, dass es sich bei der Arbeit von Hindrichsen/Schultz um eine bikulturelle Studie handelt, die zwar Unterschiede im dänische-deutschen Kontext aufzeigen kann, aber nicht darüber Auskunft gibt, ob der Werbestil der heilen Welt beispielsweise auch in Spanien, Frankreich oder Polen ein nationalkulturelles Kennzeichen der Werbung in diesen Ländern sein könnte.

\section{Literatur}

Averbeck, Stefanie (2002): „Lippmann: Public Opinion“. In: Holtz-Bacha, Christina/Kutsch, Arnulf (eds.): Schlüsselwerke für die Kommunikationswissenschaft. Wiesbaden, Westdeutscher Verlag: 273-276.

Backhaus, Klaus/Voeth, Markus (2010): Internationales Marketing. Stuttgart: SchäfferPoeschel.

Bendel, Sylvia (2008): „Werbestrategien hinterfragen statt reproduzieren - Plädoyer für eine kritische Wissenschaft“. In: Held, Gudrun/Bendel, Sylvia (eds.): Werbung - grenzenlos. Multimodale Werbetexte im interkulturellen Vergleich. Frankfurt a. M. etc., Lang: 229244.

Bergengruen, Werner (1950): Die heile Welt. Gedichte. München: Nymphenburger Verlagshandlung.

Bohnsack, Ralf (2003): „Heidi““. In: Ehrenspeck, Yvonne/Schäffer, Burkhard (eds.): Filmund Fotoanalyse in der Erziehungswissenschaft. Ein Handbuch. Opladen, Leske + Budrich: 109-120.

Bußmann, Hadumod (2002): Lexikon der Sprachwissenschaft. Stuttgart: Kröner.

Christensen, Lars Thøger (2001): Reklame i selvsving. Frederiksberg: Samfundslitteratur.

Christensen, Lars Thøger/Cheney, George (2000): "Self-Absorption and Self-Seduction in the Corporate Identity Game”. In: Schultz, Majken/Hatch, Mary Jo/Holten Larsen, Mogens (eds.): The Expressive Organization. Oxford, Oxford University Press: 246-270.

de Mooij, Marieke (1998): Global Marketing and Advertising. Understanding Cultural Paradoxes. Thousand Oaks/London/New Delhi: Sage Publications.

de Mooij, Marieke (2001): "The Impact of Culture on Advertising”. In: Kloss, Ingomar (ed.): Advertising Worldwide. Advertising Conditions in Selected Countries. Berlin, Springer: 124.

de Mooij, Marieke (2010): Global Marketing and Advertising. Understanding Cultural Paradoxes. 3. Ausgabe. Thousand Oaks/London/New Delhi: Sage Publications. 
Demarmels, Sascha (2008): „Die Produktwerbung von Valserwasser - eine Globalisierungsgeschichte?“ In: Held, Gudrun/Bendel, Sylvia (eds.): Werbung - grenzenlos. Multimodale Werbetexte im interkulturellen Vergleich. Frankfurt a. M. etc., Lang: 191-209.

Dreßler, Raphaela (2008): „Vom Patriarchat zum androgynen Lustobjekt - 50 Jahre Männer im stern“. In: Holtz-Bacha, Christina (ed.): Stereotype? Frauen und Männer in der Werbung. Wiesbaden, VS Verlag für Sozialwissenschaften: 124-154.

Femers, Susanne (2007): Die ergrauende Werbung: Altersbilder und werbesprachliche Inszenierungen von Alter. Wiesbaden: VS Verlag für Sozialwissenschaften.

Fröhlich, Romy (2008): „Werbung in Deutschland - auf dem Weg zum Frauenberuf?“. In: Holtz-Bacha, Christina (ed.): Stereotype? Frauen und Männer in der Werbung. Wiesbaden, VS Verlag für Sozialwissenschaften: 14-39.

Friestad, Marian/Wright, Peter (1994): “The Persuasion Knowledge Model: How People Cope with Persuasion Attempts". Journal of Consumer Research 21/1: 1-31.

Gasteiger, Nepomuk (2008): „Vom manipulierbaren zum postmodernen Konsumenten. Das Bild des Verbrauchers in der westdeutschen Werbung und Werbekritik, 1950-1990“. Archiv für Kulturgeschichte 90/1: 129-157.

Hars, Wolfgang (2002): Nichts ist unmöglich! Lexikon der Werbesprüche. München: Piper.

Hindrichsen, Anne/Schultz, Birgit (2004): Tyske, versionerede tv-spots - en analyse af hvorfor danskere ikke bryder sig om tyske, versionerede tv-spots for slik og chokolade. Unveröffentlichte Master-Arbeit, Wirtschaftsuniversität Aarhus.

Hoffmann, Michael (1999): „Thesen zur Varietätenlinguistik“. Zeitschrift für Germanistische Linguistik 27/3: 309-321.

Hoffmann, Michael (2012): „Werbekommunikation stilistisch“. In: Janich, Nina (ed.): Werbekommunikation. Sprachwissenschaftliche und interdisziplinäre Zugänge. Tübingen, Narr/Francke/Attempto: 179-196.

Holtz-Bacha, Christina (2008): „Köcheln auf kleiner Flamme. Frauen und Männer in der Werbung“. In: Holtz-Bacha, Christina (ed.): Stereotype? Frauen und Männer in der Werbung. Wiesbaden, VS Verlag für Sozialwissenschaften: 5-12.

Janich, Nina (1997): „Wenn Werbung mit Werbung Werbung macht ... Ein Beitrag zur Intertextualität“". Muttersprache 107/4: 297-309.

Janich, Nina (ed.) (2012): Werbekommunikation. Sprachwissenschaftliche und interdisziplinäre Zugänge. Tübingen: Narr/Francke/Attempto.

Janich, Nina (2013): Werbesprache. Ein Arbeitsbuch. Tübingen: Narr.

Kleinsteuber, Hans J. (1991): „Was sind Feindbilder und Stereotype? Stereotype, Images und Vorurteile - Die Bilder in den Köpfen der Menschen“. In: Trautmann, Günter (ed.): Die häßlichen Deutschen. Deutschland im Spiegel der westlichen und östlichen Nachbarn. Darmstadt, Wissenschaftliche Buchgesellschaft: 60-68.

Kloss, Ingomar (ed.) (2001a): Advertising Worldwide. Heidelberg: Springer.

Kloss, Ingomar (2001b): “Advertising in Germany”. In: Kloss, Ingomar (ed.): Advertising Worldwide. Heidelberg, Springer: 118-148.

Kroeber-Riel, Werner/Weinberg, Peter/Gröppel-Klein, Andrea (2009): Konsumentenverhalten. München: Vahlen.

Lippmann, Walter (1922): Public opinion. New York: Macmillan. 
Moritz, Peter (1997): „Der Schock des Unverständlichen. Medienethik und Medienkultur im Kreuzfeuer medial arrangierter Provokation“. Medienimpulse Dezember 97: 49-60.

Motschenbacher, Heiko (2006): „Men and Women Like Different Things“? - Doing Gender als Strategie der Werbesprache. Marburg: Tectum.

Motschenbacher, Heiko (2008): „Werbesprachliche Genderstilisierung für globale Zeitschriften-Communities“. In: Held, Gudrun/Bendel, Sylvia (eds.): Werbung - grenzenlos. Multimodale Werbetexte im interkulturellen Vergleich. Frankfurt a. M. etc., Lang: 57-76.

Müller, Wendelin (1997): Interkulturelle Werbung. Heidelberg: Physica.

Müller, Stefan/Gelbrich, Katja (2014a): Interkulturelles Marketing. München: Vahlen.

Müller, Stefan/Gelbrich, Katja (2014b): Interkulturelle Kommunikation. München: Vahlen.

Nielsen, Martin (2007): „Die -tainment-Welle - unaufhaltsame Unterhaltung? Aspekte des Advertainment in der Informationsgesellschaft". In: Klemm, Michael/Jakobs, Eva-Maria (eds.): Das Vergnügen in und an den Medien. Interdisziplinäre Perspektiven. Frankfurt a. M. etc., Lang: 67-84.

Nielsen, Martin (2016): „Fachspezifische Stereotype der Werbewirtschaft bei der Kampagnenadaption im dänisch-deutschen Kontext"“. In: Hallsteinsdóttir, Erla et al. (eds.): Perspektiven der Stereotypenforschung. Frankfurt a. M., Lang: 123-143.

Reimann, Sandra (2003): „Mit Liebe zum Detail wie immer das Erlebnis vollendeter Kaffeekultur! Sprach- und Zeitgeschichte im Spiegel von Dallmayr-Werbespots aus fünf Jahrzehnten“. Germanistische Linguistik 169/170: 197-216.

Reinhardt, Dirk (1993): Von der Reklame zum Marketing. Geschichte der Wirtschaftswerbung in Deutschland. Berlin: Akademie Verlag.

Ruppert, Wolfgang (2000): „Zur Konsumwelt der 60er Jahre“. In: Schildt, Axel/Siegfried, Detlef/Lammers, Karl Christian (eds.): Dynamische Zeiten. Die 60er Jahre in den beiden deutschen Gesellschaften. Hamburg, Christians: 752-767.

Schmidt, Siegfried J./Spieß, Brigitte (1994a): „Geschichte der Fernsehwerbung in der Bundesrepublik Deutschland: Eine Skizze“. In: Erlinger, Hans-Dieter/Foltin, Hans-Dietrich (eds.): Geschichte des Fernsehens in der Bundesrepublik Deutschland. Bd. 4. Unterhaltung, Werbung und Zielgruppenprogramme. München, Fink: 187-242.

Schmidt, Siegfried J./Spieß, Brigitte (1994b): Die Geburt der schönen Bilder. Fernsehwerbung aus der Sicht der Kreativen. Opladen: Westdeutscher Verlag.

Schoenen, Judith (2008): Das Image der Frau. Wege zu einem neuen Selbstbild. Opladen/Farmington Hills: Budrich.

Schweiger, Günter/Schrattenecker, Gertraud (2001): Werbung. 5. Auflage. Stuttgart: Lucius $\&$ Lucius.

Seeßlen, Georg (1992): „Die Werbung sagt immer die Wahrheit“. Medien Concret 1/92: 1219.

Siegert, Gabriele/Brecheis, Dieter (2010): Werbung in der Medien- und Informationsgesellschaft. Wiesbaden: VS Verlag für Sozialwissenschaften.

Six, Bernd (1999): „Vorurteil““. In: Asanger, Bernd/Wenninger, Gerd (eds.): Handwörterbuch Psychologie. Weinheim, Beltz: 828-830.

Thomsen, Christian W. (1998): „Strategien, Trends und Ästhetik deutscher Fernsehwerbung 1997“. In: Pethig, Rüdiger/Blind, Sofia (eds.): Fernsehfinanzierung. Ökonomische, rechtliche und ästhetische Perspektiven. Opladen/Wiesbaden, Westdeutscher Verlag: 192-208. 
Vennemann, Angela/Holtz-Bacha, Christina (2008): „Mehr als Frühjahrsputz und Südseezauber? Frauenbilder in der Fernsehwerbung und ihre Rezeption“. In: Holtz-Bacha, Christina (ed.): Stereotype? Frauen und Männer in der Werbung. Wiesbaden, VS Verlag für Sozialwissenschaften: 76-106.

Wilk, Nicole (2008): „Die ges(ch)ichtslose Frau - Überlegungen zum Verlust von weiblichen Vorbildern in der Werbung“. In: Holtz-Bacha, Christina (ed.): Stereotype? Frauen und Männer in der Werbung. Wiesbaden, VS Verlag für Sozialwissenschaften: 51-75.

\section{Korpus}

Amazon (s. a.): „Nivea Visage Aqua Sensation Anti-Shadow Eye Cream 15ml“. www.amazon.de/Nivea-Visage-Sensation-Anti-Shadow-Cream/dp/B0026L7EAU [21.03.2016].

Dr. BEST (s. a.): „Werbung mit Dr. James Best: Das frühe Gesicht der Marke Dr.BEST ${ }_{\mathbb{B}}$ “. www.dr-best.de/geschichte.html [21.03.2016].

Flensburger (2011): „Flensburger Pilsener Werbung“. Veröffentlicht auf YouTube am 3. Oktober 2011. www.youtube.com/watch? $\mathrm{v}=\mathrm{eQ} 3 \mathrm{ky} 6 \mathrm{dCC}-4$ [21.03.2016].

Flensburger Brauerei (s. a.): „Werbung“. www.flens.de/brauerei/werbung/ [15.03.2016].

fluege.de (s. a.). www.fluege.de [21.05.2015].

Henkel Lifetimes (2015): „Streifen auf Tassenböden entfernen“. Veröffentlicht auf YouTube am 25. November 2015. www.youtube.com/watch?v=WB224RA8CRM [21.03.2016].

Kinder (2012): „Werbespot für Kinder-Überraschungseier“. Veröffentlicht auf YouTube am 18. Dezember. www.youtube.com/watch?v=4hjUVShDhCY [21.03.2016].

Lindt (2015): „Lindt GOLDHASE“. Veröffentlicht auf YouTube am 18. Februar 2015. www.youtube.com/watch?v=C1FhCuzuzUo [21.03.2016].

LRF = Light Rock Fishing (2012): „Marukyu Japan Advertising“. http://light.rockfishing.co.uk/2012/09/marukyu-japan-advertising [21.03.2016].

Merci (2015): „Merci Werbung 2015-01“. Veröffentlicht auf YouTube am 18. Januar 2015. www.youtube.com/watch?v=eyCt816u5MI [21.03.2016].

Nestlé (s. a.): „AFTER EIGHT®“. Www.nestle.de/marken/alle-marken/after-eight [21.03.2016].

Nordzucker (s. a.): „SweetFamily für die süßen Augenblicke im Leben“. www.nordzucker.de/verbraucher/marken/sweetfamily.html [21.03.2016].

Opel (2015): „The new Opel KARL“. Veröffentlicht auf YouTube am 24. September 2015. www.youtube.com/watch?v=SNEm4Z4HYA8 [21.03.2016].

Saal, Marko (2013): „Wie Sixt die Liberalen veralbert“. Horizont, 25. September 2013. www.horizont.net/marketing/nachrichten/Mehr-Sitze-als-die-FDP-Wie-Sixt-die-

Liberalen-veralbert-116926 [21.03.2016].

Toffifee (2012): „Toffifee Werbung“. Veröffentlicht auf YouTube am 12. März 2012. www.youtube.com/watch?v=Ebqq4O92yGo [21.3.2016].

Werthers (2012): „Werthers Echte 1992“. Veröffentlicht auf YouTube am 16. September 2012. www.youtube.com/watch?v=6R0j4c5ipgI [21.03.2016]. 